\title{
CDKN3 promotes cell proliferation, invasion and migration by activating the AKT signaling pathway in esophageal squamous cell carcinoma
}

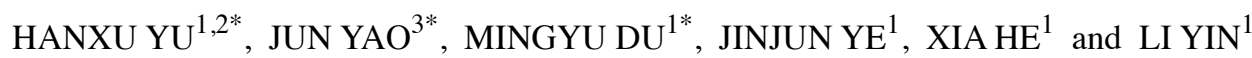 \\ ${ }^{1}$ Department of Radiotherapy, The Affiliated Cancer Hospital of Nanjing Medical University, \\ Jiangsu Cancer Hospital, Jiangsu Institute of Cancer Research, Nanjing, Jiangsu 210000; ${ }^{2}$ Department of Radiotherapy, \\ Lianshui County People's Hospital, Lianshui, Jiangsu 223001; ${ }^{3}$ Department of Radiotherapy, \\ Yancheng Second People's Hospital, Yancheng, Jiangsu 22400, P.R. China
}

Received April 8, 2019; Accepted September 26, 2019

DOI: $10.3892 / \mathrm{ol} .2019 .11077$

\begin{abstract}
In China, esophageal squamous cell carcinoma (ESCC), capable of direct invasion and early metastasis, exhibits high mortality. Identification of the molecular basis driving ESCC progression and development of new diagnostic biomarkers are urgently needed. Cyclin-dependent kinase inhibitor 3 (CDKN3) performs crucial roles in the modulation of tumor development. The present study aimed to explore the functions and underlying mechanism of CDKN3 in regulating ESCC cell proliferation and invasion. The expression levels of CDKN3 in ESCC cells were evaluated by reverse transcription-quantitative PCR. Cell counting kit-8 and colony forming assays were used to evaluate cell viability. Wound-healing assay was performed to explore cell migration. Transwell invasion analysis was conducted to investigate the invasive capacity of ESCC cells. Protein levels were detected by western blot assay. The results demonstrated that the expression of CDKN3 was significantly upregulated in ESCC tissues, as predicted using the UALCAN and Gene Expression Omnibus databases. PCR and western blot assays confirmed that CDKN3 was upregulated in ESCC cell lines. Functional assays revealed that CDKN3 knockdown with small interfering RNA decreased the ability of ESCC cells to proliferate, invade and migrate and suppressed G1/S transition. Further mechanistic analyses demonstrated that CDKN3
\end{abstract}

Correspondence to: $\mathrm{Dr} \mathrm{Xia} \mathrm{He}$ or Dr Li Yin, Department of Radiotherapy, The Affiliated Cancer Hospital of Nanjing Medical University, Jiangsu Cancer Hospital, Jiangsu Institute of Cancer Research, 42 Bai Zi Ting Road, Nanjing, Jiangsu 210000, P.R. China E-mail: hexiabm@163.com

E-mail: yinli_2012@126.com

*Contributed equally

Key words: esophageal squamous cell carcinoma, cyclin-dependent kinase inhibitor 3, proliferation, invasion promoted cell proliferation and invasion by activating the AKT signaling pathway in ESCC cells. To the best of our knowledge, the present study is the first to identify the functions of CDKN3 in ESCC and provide evidence that CDKN3 regulates tumor progression by activating the AKT signaling pathway. Therefore, CDKN3 may serve as a potential effective therapeutic target for ESCC treatment.

\section{Introduction}

Esophageal cancer (EC) is one of the most common malignancies that leads to high mortality and poor prognosis worldwide (1). Approximately 572,034 new diagnosed cases and 508,585 deaths occurred in 2018 worldwide (2). In China, esophageal squamous cell carcinoma (ESCC), which is capable of direct invasion and early metastasis, is the most prevalent pathological type of EC; ESCC accounted for $>90 \%$ of EC cases in 2011 in China $(3,4)$. Despite advances in diagnostic technologies and treatment, the overall survival of patients with ESCC remains poor (5-7). Thus, identifying the mechanistic basis of ESCC progression and developing novel therapeutic targets for patients with ESCC is urgently needed.

Cyclin-dependent kinase inhibitor 3 (CDKN3) serves crucial roles in the cell cycle and proliferation (8-10). CDKN3 performs its functions by binding to cyclin proteins, which results in dephosphorylation of CDK1 and CDK2 proteins and inhibition of cell cycle progression (11-13). The dynamic expression of CDKN3 and its oncogenic role have been widely explored in various types of cancer (14-16). A previous study has reported that CDKN3 is upregulated in cervical cancer and associated with reduced survival time, indicating that it may be a potential biomarker for patients with cervical cancer (15). Zang et al (16) have demonstrated that CDKN3 is expressed at high levels in lung adenocarcinoma and is associated with poor survival outcomes. Silencing CDKN3 suppresses cell proliferation and tumorigenesis in nasopharyngeal carcinoma by regulating the expression of p27 (17). Deng et al (18) demonstrated that CDKN3 exhibits a high expression in breast cancer cell lines, thus promoting apoptosis and inhibiting cell migration. Xu et al (19) used pathway analysis to explore the 
differentially expressed genes in ESCC; the results indicated that CDKN3 is upregulated in ESCC and functions as a key gene in signal transduction networks (including PI3K-Akt signaling pathway, and cell cycle). Although a number of studies have demonstrated that CDKN3 expression is upregulated in ESCC, limited information is available regarding the function and mechanism of CDKN3 in ESCC development.

In the present study, database search was used to determine the levels of CDKN3 expression in ESCC tissues and cells. Functional experiments were also employed to explore the functions of CDKN3 in ESCC cells.

\section{Materials and methods}

Cell lines. ESCC cell lines EC-1 (cat. no. BNCC339894, 1), EC-7 (named KYSE510; cat. no. BNCC342111), Eca-109 (cat. no. BNCC337687) and TE-1 (cat. no. BNCC100151) were obtained from the BeNa Culture Collection. An epithelial cell line Het1A (cat. no. ATCC CRL-2692) was obtained from the American Type Culture Collection. ESCC cells were cultured in RPMI-1640 medium (Gibco; Thermo Fisher Scientific, Inc.) containing $10 \%$ fetal bovine serum at $37^{\circ} \mathrm{C}$ with $5 \% \mathrm{CO}_{2}$.

Cell transfection. Small interfering (si)RNAs si-CDKN3 and si-NC were obtained from Guangzhou RiboBio Co., Ltd. The sequences were as follows: CDKN3 siRNA 1, 5'-GTGGAA TTATCACCCATCA-3'; CDKN3 siRNA 2, 5'-CTGCTTGTC TCCTACTATA-3'; si-NC, 5'-GGCUCUAGAAAAGCCUAU GC-3'. For transfection, Eca-109 and TE-1 cells were cultured in 6 -well plates $\left(1.5 \times 10^{5}\right.$ cells/well $)$ and transfected with $2 \mu \mathrm{g}$ si-CDKN3 or $2 \mu \mathrm{g}$ si-NC using Lipofectamine ${ }^{\circledR}$ iMAX (Invitrogen; Thermo Fisher Scientific, Inc.). Following transfection, Eca-109 and TE-1 cells were cultured at $37^{\circ} \mathrm{C}$ with $5 \% \mathrm{CO}_{2}$ for $48 \mathrm{~h}$ prior to subsequent experiments. Reverse transcription-quantitative PCR (RT-qPCR) and western blotting were used to determine transfection efficiency.

$R T$ - $q P C R$ assay. RNA was extracted from Eca-109 and TE-1 cells using TRIzol ${ }^{\circledR}$ reagent (Invitrogen; Thermo Fisher Scientific, Inc.). cDNA was reverse-transcribed from RNA using the PrimeScript ${ }^{\mathrm{TM}}$ High Fidelity RT-PCR kit (Takara Biotechnology Co., Ltd.) for mRNA expression analysis. The reaction conditions for reverse transcription were $37^{\circ} \mathrm{C}$ for $15 \mathrm{~min}$ and $85^{\circ} \mathrm{C}$ for $5 \mathrm{sec}$. RT-qPCR was conducted using SYBR $^{\circledR}$-Green (Applied Biosystems; Thermo Fisher Scientific, Inc.) on an ABI7500 real-time PCR instrument (Applied Biosystems; Thermo Fisher Scientific, Inc.). The thermocycling conditions were as follows: $95^{\circ} \mathrm{C}$ for $30 \mathrm{sec}$, followed by 40 cycles of $95^{\circ} \mathrm{C}$ for $15 \mathrm{sec}$ and $60^{\circ} \mathrm{C}$ for $1 \mathrm{~min}$. The specific primers for $\mathrm{CDKN} 3$ and $\beta$-actin used were: $\mathrm{CDKN} 3$ forward, 5'-GTCCCAAACCTTCTGGATCTCTAC-3' and reverse, 5'-AGCTCTTCCATTATTTCACAGCAG-3'; $\beta$-actin forward, 5'-GGACTTCGAGCAAGAGATGG-3' and reverse, 5'-AGC ACTGTGTTGGCGTACAG-3'. The relative expression of CDKN3 was calculated using the $2^{-\Delta \Delta \mathrm{Cq}}$ method (20).

Cell proliferation assays. At $48 \mathrm{~h}$ post-transfection, $3 \times 10^{3}$ transfected cells/well (Eca-109 and TE-1 cells) were inoculated into 96 -well plates and incubated at $37^{\circ} \mathrm{C}$ with $5 \% \mathrm{CO}_{2}$. Cell Counting Kit-8 (Dojindo Molecular Technologies, Inc.) solution $(10 \mu \mathrm{l})$ was added to each well for $1 \mathrm{~h}$ at $37^{\circ} \mathrm{C}$ and absorbance was recorded at $450 \mathrm{~nm}$ on days 1,2, 3 and 4. For colony formation assay, transfected ESCC cells (100 cells/well) were seeded in a 6-well plate and incubated at $37^{\circ} \mathrm{C}$ with $5 \%$ $\mathrm{CO}_{2}$ for 10 days. The colonies were fixed with $4 \%$ formaldehyde for $30 \mathrm{~min}$ and stained with $0.2 \%$ crystal violet for $2 \mathrm{~h}$ at room at room temperature. Colonies containing $>50$ cells were counted using a light microscope (magnification, x200) using the ImageJ software (National Institutes of Health).

Wound-healing assay. Wound-healing assay was used to detect the migration of Eca-109 and TE-1 cells. ESCC cells were transfected with siCDKN3 or si-NC in six-well plates and cultured for $48 \mathrm{~h}$ until $90 \%$ confluency was reached. A 200- $\mu 1$ pipette tip was used to create an artificial wound. The cells were washed with PBS and cultured in serum-free RPMI-1640 medium. Wound closure was visualized at 0 and $24 \mathrm{~h}$ using an inverted light microscope (magnification, x200). The distance between the edges of the wound was calculated as previously described.

Invasion assay. Matrigel assay was used to determine cell invasiveness. Transfected Eca-109 and TE-1 cells $\left(\sim 2 \times 10^{4}\right)$ were harvested, resuspended in 200- $\mu 1$ serum-free RPMI-1640 medium and added into the upper Transwell chamber pre-coated with Matrigel. The lower part of the chamber was filled with medium containing $20 \%$ FBS. Following 12 or $24 \mathrm{~h}$ of incubation, the cells on the top surface of the filters were removed from the upper chamber using a cotton swab. The cells that invaded through the Matrigel membrane were fixed with $4 \%$ paraformaldehyde and stained with $0.5 \%$ crystal violet at $37^{\circ} \mathrm{C}$ for $2 \mathrm{~h}$ at room temperature. Cell images from five randomly fields were selected and counted using a light microscope with x200 magnification.

Flow cytometric analysis. Following transfection, $1 \times 10^{5}$ cells (Eca-109 and TE-1 cells) were seeded onto 6-well plates and cultured for $24 \mathrm{~h}$ at $37^{\circ} \mathrm{C}$. The cells were harvested and fixed with $70 \%$ ethanol for $24 \mathrm{~h}$ at $-20^{\circ} \mathrm{C}$. The processed cells were stained with propidium iodide (PI) staining solution (BD Pharmingen) at $37^{\circ} \mathrm{C}$ for $10 \mathrm{~min}$. Cell cycle distribution was analyzed by FACScalibur flow cytometer (BD Biosciences) and the ModFit LT (version 3.2) software. All experiments were repeated three times.

Western blot analysis. Transfected Eca-109 and TE-1 cells were suspended and lysed with RIPA buffer (Beyotime Institute of Biotechnology). Protein was extracted from the cells and quantified using a Bicinchoninic Acid kit (Beyotime Institute of Biotechnology). Protein (15 $\mu \mathrm{g} / \mathrm{lane}$ ) was separated using $10 \%$ SDS-PAGE and transferred onto a PVDF membrane (EMD Millipore). The membrane was subsequently blocked with 5\% skimmed milk in Tris-buffered saline containing $0.1 \%$ Tween-20 (TBST) for $2 \mathrm{~h}$ at $37^{\circ} \mathrm{C}$ and incubated at $4^{\circ} \mathrm{C}$ overnight with the following antibodies: Anti-CDKN3 (1:1,000; cat. no. ab175393; Abcam), anti-AKT (1:1,000; cat. no. ab179463; Abcam), anti-p-AKT (1:1,000; cat. no. ab38449; Abcam) and anti-cyclinD1 (1:1,000; cat. no. 2922; Cell Signaling Technology, Inc.). Anti-p27 (1:1,000; cat. no. 3686S; Cell Signaling Technology, Inc.) and 
A Expression of CDKN3 in ESCA based on Sample types

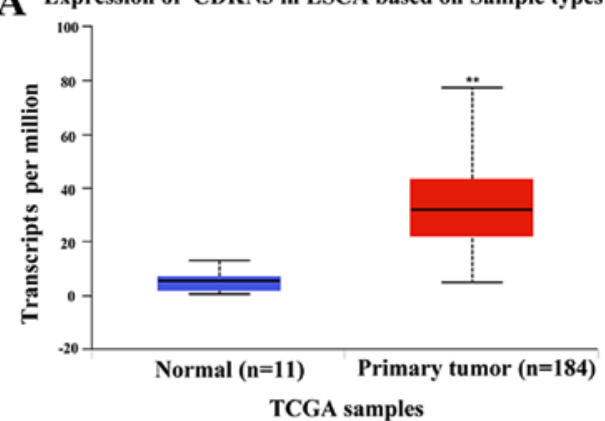

C Expression of CDKN3 in ESCA based on individual

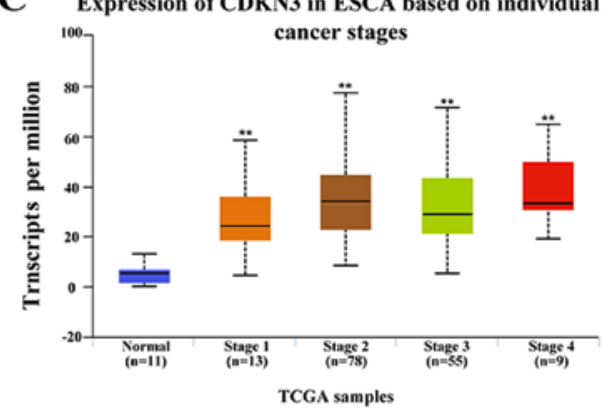

$\mathbf{E}$

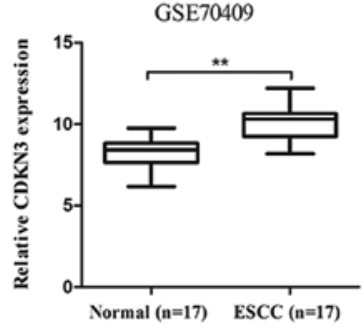

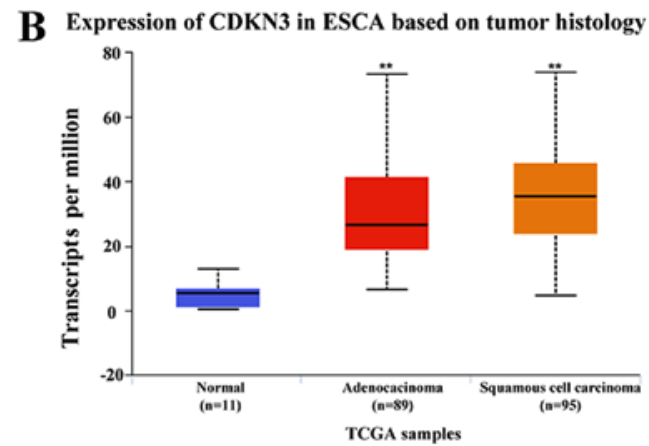

D

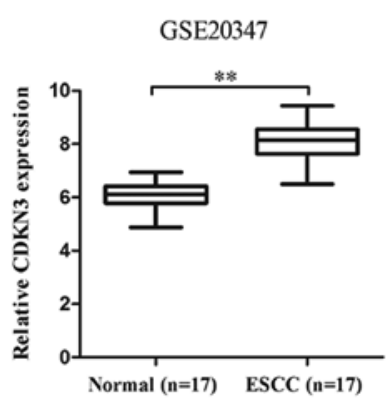

Figure 1. CDKN3 expression is upregulated in ESCC. (A) CDKN3 expression was significantly higher in esophageal cancer (n=184) compared with normal esophageal tissues $(\mathrm{n}=11)$ in samples obtained from the UALCAN database. (B) CDKN3 expression in normal esophageal tissues ( $\mathrm{n}=11)$, esophageal adenocarcinoma ( $\mathrm{n}=89$ ) and ESCC $(\mathrm{n}=95)$. (C) CDKN3 expression in normal esophageal tissues and esophageal cancer tissues obtained from patients with different stages of ESCA. (D) CDKN3 expression in $17 \mathrm{ESCC}$ and 17 normal esophageal tissues in the GEO data set GSE20347. (E) CDKN3 expression in 17 ESCC and 17 normal esophageal tissues using the GEO data set GSE70409. ** $\mathrm{P}<0.01$ vs. normal tissues. CDKN3, cyclin-dependent kinase inhibitor 3; ESCC, esophageal squamous cell carcinoma; ESCA, esophageal carcinoma; TCGA, The Cancer Genome Atlas.

$\beta$-actin (1:2,000; cat. no. 4970; Cell Signaling Technology, Inc.) were used as loading controls. Following three washes with TBST, the membranes were incubated with horseradish peroxidase-conjugated secondary goat anti-rabbit immunoglobulin G antibody (1:1,000; cat. no. 7074; Cell Signaling Technology, Inc.) for $1 \mathrm{~h}$ at $37^{\circ} \mathrm{C}$. Immunoreactive bands were visualized using ECL reagent (Beyotime Institute of Biotechnology).

Bioinformatics analysis. The Cancer Genome Atlas (TCGA) analysis was performed using the UALCAN website (http://ualcan.path.uab.edu/index.html) as previously described (21). Microarray data of mRNA expression from the Gene Expression Omnibus (GEO; http://www.ncbi.nlm. nih.gov/geo) data sets GSE20347 and GSE70409 $(22,23)$ were downloaded and analyzed by DESeq packages in R (http://www. bioconductor.org/packages/release/bioc/html/DESeq.html). The list of genes co-expressed with CDKN3 obtained from the UALCAN database were annotated for Gene Ontology (GO) and Kyoto Encyclopedia of Genes and Genomes (KEGG) pathway enrichment analyses using DAVID (https://david. ncifcrf.gov/).
Statistical analysis. All experiments were repeated three times and data are presented as the mean \pm standard deviation. Data were processed using SPSS 20.0 (IBM Corp.) and GraphPad Prism 7.0 (GraphPad Software, Inc.). Student's t-test was used to compare the differences between two groups. Comparisons among multiple groups were analyzed using one-way ANOVA followed by Tukey's post hoc test. $\mathrm{P}<0.05$ was considered to indicate a statistically significant difference.

\section{Results}

CDKN3 is upregulated in ESCC. To determine the role of CDKN3 in ESCC, an interactive website UALCAN (http://ualcan.path.uab.edu), which is based on TCGA gene expression data, was used to analyze CDKN3 expression in ESCC. The results demonstrated that CDKN3 was significantly upregulated in esophageal carcinoma (ESCA) tissues compared with normal samples (Fig. 1A). The difference in CDKN3 expression between esophageal adenocarcinoma (EAC) and ESCC was not significant $(\mathrm{P}=0.06)$; CDKN3 expression levels in EAC and ESCC were higher compared with those in normal samples (Fig. 1B). The expression of 
A

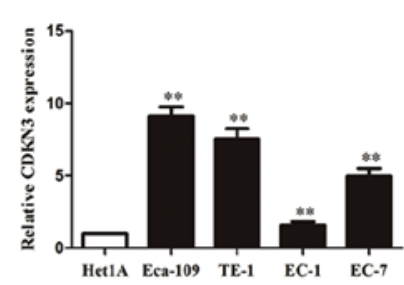

$\mathrm{C}$

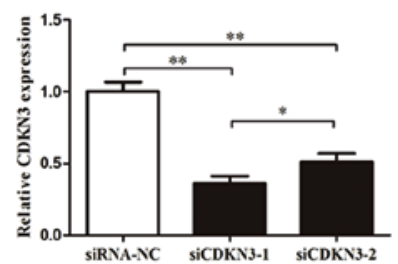

$\mathrm{B}$

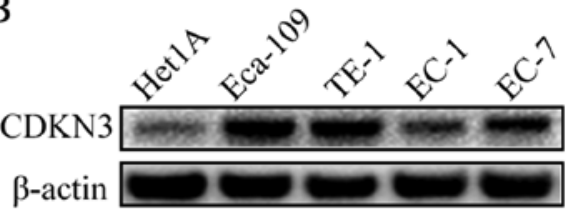

$\mathrm{D}$

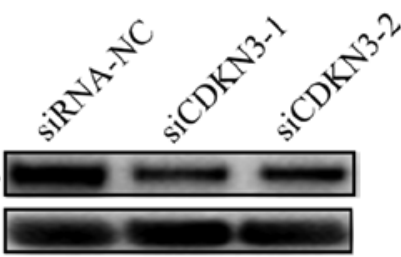

Figure 2. CDKN3 expression is upregulated in ESCC cell lines and effectively knocked down by siRNA. (A and B) PCR and western blot assays demonstrated that CDKN3 was upregulated in ESCC cell lines. (C and D) PCR and western blot assays demonstrated that CDKN3 was effectively knocked down by siRNA. ${ }^{* *} \mathrm{P}<0.01$ vs. control (Het1A or siRNA-NC). CDKN3, cyclin-dependent kinase inhibitor 3; ESCC, esophageal squamous cell carcinoma; siRNA, small interfering RNA; NC, negative control; siCDKN3, cells transfected with siRNA targeting CDKN3.

A
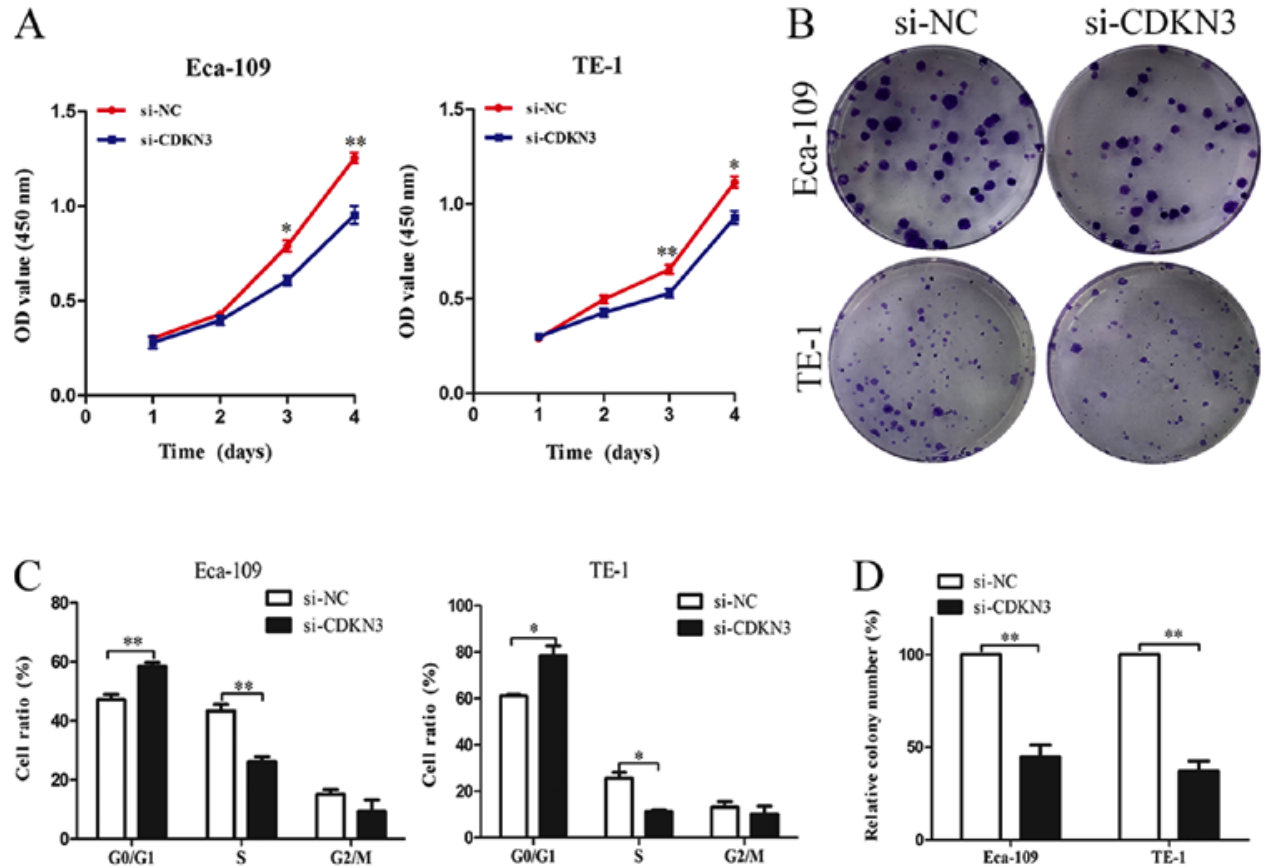

Figure 3. CDKN3 knockdown suppresses the proliferation, migration and invasion of ESCC cells. (A) Cell counting kit-8 assay demonstrated that CDKN3 knockdown suppressed the cell proliferative ability. (B and D) Colony formation assay indicated that CDKN3 knockdown inhibited the proliferation of ESCC cells. (C) The results of cell cycle showed that knock down of CDKN3 increased the cells in G0/G1 phase. ${ }^{*} \mathrm{P}<0.05$, ${ }^{* *} \mathrm{P}<0.01$ vs. si-NC. CDKN3, cyclin-dependent kinase inhibitor 3; ESCC, esophageal squamous cell carcinoma; si-NC, cells transfected with the negative control small interfering RNA; siCDKN3, cells transfected with small interfering RNA targeting CDKN3; OD, optical density.

CDKN3 in patients with esophageal cancer, according to their stage, is shown in Fig. 1C. The data from the GEO database revealed higher CDKN3 expression in ESCC compared with that in normal esophageal samples in the GSE20347 and GSE70409 datasets, which confirmed that CDKN3 was upregulated in ESCC.

CDKN3 is upregulated in ESCC cell lines and effectively knocked down by siRNA. CDKN3 expression in ESCC was examined using qPCR and western blot analyses. The expression levels of CDKN3 were significantly upregulated in EC-1,
EC-7, Eca-109 and TE-1 cells compared with those in normal esophageal epidermal cells (Het1A). Subsequent experiments were performed to determine whether the two siRNAs effectively knocked down CDKN3 expression in ESCC cells. Transfection with siRNAs reduced the mRNA and protein expression levels of CDKN3 compared with the negative control, and siCDKN3-1 exhibited a stronger inhibitory effect compared with siCDKN3-2 (Fig. 2C and D). These results suggested that CDKN3 was upregulated in ESCC cells, and siCDKN3-1 was an effective approach for silencing CDKN3 expression. 

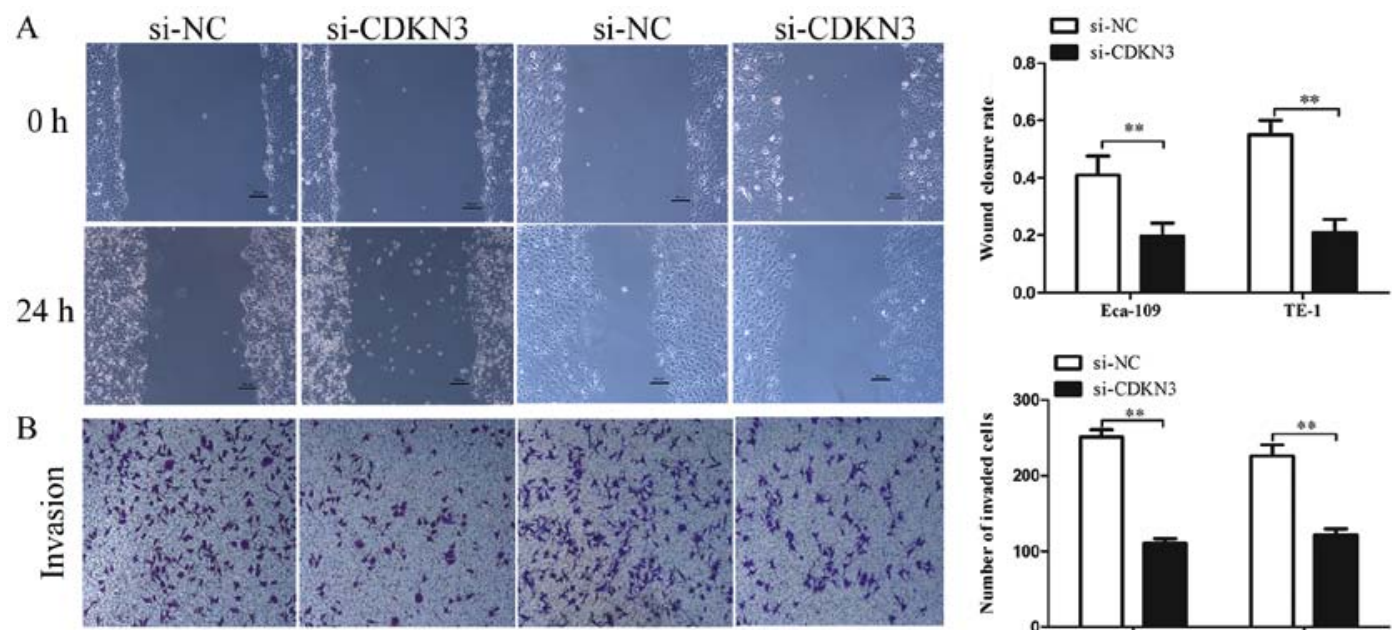

Eca-109

TE-1

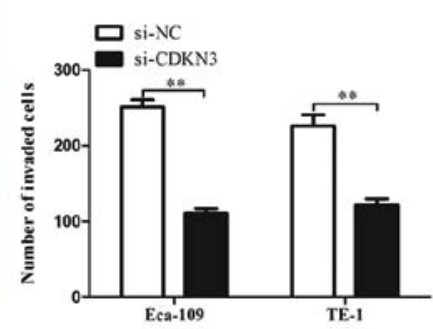

Figure 4. CDKN3 knockdown suppresses the migration and invasion of ESCC cells. (A) The wound-healing and assay indicated that CDKN3 knockdown significantly reduced the migration of ESCC cells. Magnification, x200; scale bars, $200 \mu \mathrm{m}$. (B) The Matrigel assay demonstrated that CDKN3 knockdown significantly inhibited the invasive capacity of ESCC cells. Magnification, $\mathrm{x} 200$; scale bars, $200 \mu \mathrm{m}$. " $\mathrm{P}<0.01$. CDKN3, cyclin-dependent kinase inhibitor 3 ; ESCC, esophageal squamous cell carcinoma; si-NC, cells transfected with the negative control small interfering RNA; siCDKN3, cells transfected with small interfering RNA targeting CDKN3.
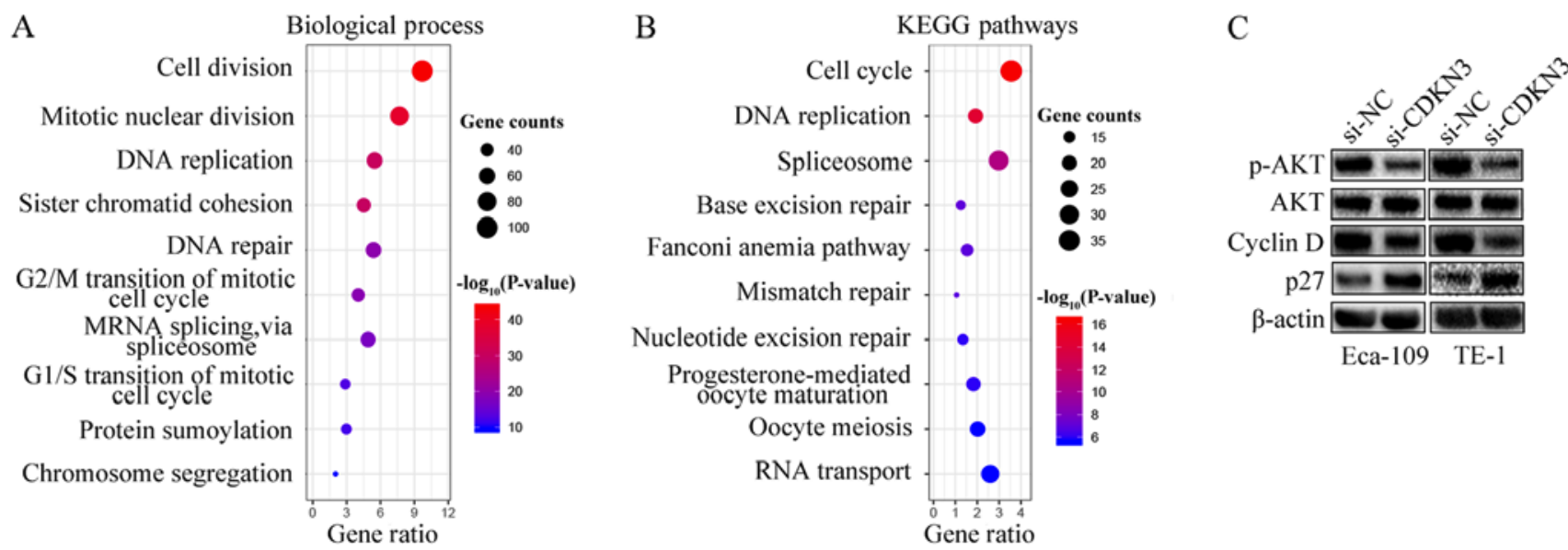

Figure 5. CDKN3 knockdown suppresses ESCC cell proliferation and invasion by inhibiting the AKT signaling pathway. (A and B) Significantly enriched Gene Ontology biological process and KEGG analyses of the genes co-expressed with CDKN3 obtained from the UALCAN database. (C) Western blot analysis demonstrated that CDKN3 knockdown reduced the protein levels of p-AKT and cyclin D. CDKN3, cyclin-dependent kinase inhibitor 3; ESCC, esophageal squamous cell carcinoma; KEGG, Kyoto Encyclopedia of Genes and Genomes; si-NC, cells transfected with the negative control small interfering RNA; siCDKN3, cells transfected with small interfering RNA targeting CDKN3.

CDKN3 knockdown suppresses ESCC cell proliferation, invasion and migration. The biological functions of CDKN3 in ESCC cell lines were characterized. CCK-8 assay revealed that ESCC cells transfected with si-CDKN3 exhibited lower cell viability compared with the control groups (Fig. 3A). The colony formation assay also demonstrated that CDKN3 knockdown significantly reduced the proliferation of ESCC cells (Fig. 3B and D). The cell cycle analysis results suggested that CDKN3 knockdown increased the proportion of cells in the G0/G1 phase, which indicated that CDKN3 knockdown may inhibit the proliferation of ESCC cells by suppressing the G1/S transition (Fig. 3C). To determine cell invasive and migratory abilities, wound-healing and Transwell invasion assays were used. The wound closure rate of ESCC cells in the si-CDKN3 groups was lower compared with that in the controls (Fig. 4A). The Transwell invasion assay revealed that silencing CDKN3 expression significantly reduced the cell invasive capability compared with the control groups (Fig. 4B). In conclusion, the results of the functional assays suggested that CDKN3 enhanced the viability, invasion and migration of ESCC cells.

CDKN3 knockdown reduces cell proliferation and invasion by inhibiting the AKT signaling pathway in ESCC. To explore the mechanisms underlying the cell proliferation, invasion and migration enhancement by CDKN3, GO and KEGG pathway enrichment analyses were performed by characterizing the genes co-expressed with CDKN3 obtained from the UALCAN database. Processes and pathways associated with the cell cycle accounted for the highest enrichment for the co-expressed genes (Fig. 5A and B). Since CDKN3 knockdown reduced ESCC cell proliferation, invasion and migration and promoted the G0/G1 phase, the expression of cell cycle 
signaling pathway-related proteins were detected by western blot analysis. The results demonstrated that si-CDKN3 treatment downregulated the levels of p-AKT and cyclin D1 and increased the levels of p27 compared with si-NC treatment; however, no differences were observed between the protein expression levels of AKT in the two groups (Fig. 5C). Thus, CDKN3 promoted cell proliferation and invasion by activating the AKT signaling pathway.

\section{Discussion}

ESCC capable of direct invasion and early metastasis is the fourth dominant cause of tumor-related mortality in China in 2015 (24). Identification of the molecular basis underlying ESCC progression and the development of novel diagnostic biomarkers is imperative. In the present study, data from two databases, UALCAN and GEO, demonstrated that the expression of CDKN3 was higher in ESCC compared with that in normal esophageal tissues. The qPCR and western blot analyses also revealed that CDKN3 was upregulated in ESCC cell lines. The functional assays demonstrated that CDKN3 knockdown decreased the capability of ESCC cells to proliferate, invade and migrate and suppressed the G1/S transition. Further analysis indicated that CDKN3 facilitated the promotion of ESCC cell proliferation, invasion and migration by activating the AKT signaling pathway.

CDKN3 is a cyclin-dependent kinase (CDK) inhibitor that serves important roles in regulating the cell cycle progression (25). CDKN3 has been reported to be involved in tumor development and progression (14-17). Chang et al (26) have demonstrated that CDKN3 is overexpressed in nasopharyngeal cancer (NPC) tissues and that high expression of CDKN3 is associated with an advanced tumor stage and poor survival; thus, CDKN3 may serve as a predictive target for NPC. In cervical cancer, the expression of CDKN3 is associated with poor survival, and silencing CDKN3 expression significantly reduces cell proliferation (15). CDKN3 knockdown also inhibits cell invasion, proliferation and adhesion and induces cell apoptosis; high CDKN3 expression predicts poor outcomes, indicating that $\mathrm{CDKN} 3$ is involved in the oncogenic transformation process (27). Xu et al (19) used pathway analysis to explore the differentially expressed genes in ESCC and identified that CDKN3 is upregulated in ESCC and functions as a key gene in signal transduction networks. The results of the present study demonstrated that CDKN3 was upregulated in ESCC tissues and cell lines compared with normal tissues and normal esophageal epidermal cells, respectively. The CCK-8 and colony formation assays indicated that si-CDKN3 treatment significantly reduced the viability of ESCC cells, and flow cytometry demonstrated that knockdown of CDKN3 promoted the G0/G1 phase compared with the negative control groups. The wound-healing and Transwell invasion assays confirmed that silencing CDKN3 expression significantly reduced cell migratory and invasive capability compared with the controls. However, a number of previous studies have reported that CDNK3 causes opposite effects on tumor development. For example, Dai et al (28) have demonstrated that CDKN3 expression decreases in hepatocellular cell carcinoma and is related to advanced tumor stage. Another study has revealed that CDKN3 inhibition exaggerates cell survival by activating the AKT pathway (24). Studies explaining the deregulation of CDKN3 in different cancer types are limited; additional experiments must be performed to elucidate the role of CDKN3 in carcinogenesis.

A list of genes co-expressed with CDKN3 obtained from the UALCAN database was analyzed in the present study to explore the potential mechanism of CDKN3 in enhancing cell proliferation, invasion and migration. The GO and KEGG results demonstrated that the biological processes and pathways associated with the cell cycle accounted for the highest enrichment of the co-expressed genes. Wang et al (17) reported that CDKN3 performed its biological functions by targeting p27 in NPC. By contrast, Dai et al (28) have demonstrated that silencing CDKN3 expression activates the AKT/p53/p21 axis. The AKT signaling pathway serves important roles in inducing the cell cycle, proliferation and invasion. Therefore, the protein levels of the vital genes involved in the AKT signaling pathway (26-28) were determined in the present study. The results demonstrated that knockdown of CDKN3 suppressed the phosphorylation of AKT and the expression of cyclin D1 and upregulated the expression of $\mathrm{p} 27$. These results are inconsistent with those of Dai et al (28). Further study is needed to confirm the mechanism of CDKN3 in different types of cancer.

The experiments performed by Wang et al (29) focused on the functions of CDKN3 in ESCA progression and chemoresistance. ESCA includes EAC and ESCC. In the present study, the role of $\mathrm{CDKN} 3$ in regulating cell proliferation, invasion and migration was explored in ESCC cells. Another study using bioinformatics analysis has identified that CDKN3 is the key gene involved in signal transduction networks (19); however, it did not explore the functions and the underlying mechanism of CDKN3 in ESCC.

Neither of the two studies investigated the role of CDKN3 in regulating ESCC cell proliferation, invasion and migration $(19,29)$. A recent study by Liu et al (30) has reported that CDKN3 promoted the ESCC cell cycle, invasion and migration by modulating the p-AKT-p53-p21-axis. The results of the present study were consistent with their study and provided further evidence that $\mathrm{CDKN} 3$ may regulate ESCC progression by activating the AKT signaling pathway.

The present study is preliminary and there were certain limitations. Only the expression levels of cyclin D1 and p27 were detected, which was not conclusive. In future studies, the expression levels of other cyclin-related proteins, such as cyclin E, p21 and cyclin B, or other proteins in the AKT pathway will be analyzed. In addition, the effects of CDKN3 on apoptosis and the functions of CDKN3 in vivo need to be determined.

In conclusion, the results of the present study demonstrated that CDKN3 was upregulated in ESCC tissues and cells compared with the control groups, and CDKN3 knockdown significantly reduced the proliferation, migration and invasion and suppressed the G1/S transition of ESCC cell. Mechanistic analysis also revealed that CDKN3 exerted its tumor-promoting effects partly by inducing the expression of AKT signaling pathway-associated proteins. These results supported the oncogenic role of CDKN3 in the tumorigenesis of ESCC and provided a potential target for ESCC treatment. 


\section{Acknowledgements}

Not applicable.

\section{Funding}

This study was supported by the National Natural Science Foundation of China (grant no. 81672989), the Six Talent Peaks Project in Jiangsu Province (grant no. LGY2016024) and the Natural Science Foundation of Jiangsu Province (grant no. H201614).

\section{Availability of data and materials}

The datasets used and/or analysed in the present study are available from the corresponding author on reasonable request.

\section{Authors' contributions}

HY and MD designed the study. HY and JY performed the experiments. MD and JJY reviewed, analyzed and interpreted the data. XH and LY developed the original concept, wrote and revised the manuscript. All authors approved the final manuscript.

\section{Ethics approval and consent to participate}

Not applicable.

\section{Patient consent for publication}

Not applicable.

\section{Competing interests}

The authors declare that they have no competing interests.

\section{References}

1. Bray F, Ferlay J, Soerjomataram I, Siegel RL, Torre LA and Jemal A: Global cancer statistics 2018: GLOBOCAN estimates of incidence and mortality worldwide for 36 cancers in 185 countries. CA Cancer J Clin 68: 394-424, 2018.

2. Bray F, Ferlay J, Soerjomataram I, Siegel RL, Torre LA and Jemal A: Global cancer statistics 2018: GLOBOCAN estimates of incidence and mortality worldwide for 36 cancers in 185 countries. CA Cancer J Clin 68: 394-424, 2018.

3. Siegel RL, Miller KD and Jemal A: Cancer statistics. 2017. CA Cancer J Clin 67: 7-30, 2017

4. Zeng H, Zheng R, Zhang S, Zuo T, Xia C, Zou X and Chen W: Esophageal cancer statistics in China, 2011: Estimates based on 177 cancer registries. Thorac Cancer 7: 232-237, 2016.

5. Pennathur A, Gibson MK, Jobe BA and Luketich JD: Oesophageal carcinoma. Lancet 381: 400-412, 2013.

6. Zhang W, Li H, Chen X, Su M, Lin R and Zou C: Phase II study of concurrent chemoradiotherapy with a modified target volumes delineation method for inoperable oesophagealcancer patients. Br J Radiol 90: 20170328, 2017.

7. Lagergren J, Smyth E, Cunningham D and Lagergren P: Oesophageal cancer. Lancet 390: 2383-2396, 2017.

8. Cress WD, Yu P and Wu J: Expression and alternative splicing of the cyclin-dependent kinase inhibitor-3 gene in human cancer. Int J Biochem Cell Biol 91: 98-101, 2017.

9. Liu D, Zhang J, Wu Y, Shi G, Yuan H, Lu Z, Zhu Q, Wu P, Lu C, GuoF, et al: $Y Y 1$ suppresses proliferation and migration of pancreatic ductal adenocarcinoma by regulating the CDKN3/MdM2/P53/P21 signaling pathway. Int J Cancer 142: 1392-1404, 2018.
10. Yu C, Cao H, He X, Sun P, Feng Y, Chen L and Gong H: Cyclin-dependent kinase inhibitor 3 (CDKN3) plays a critical role in prostate cancer via regulating cell cycle and DNA replication signaling. Biomed Pharmacother 96: 1109-1118, 2017.

11. Nalepa G, Barnholtz-Sloan J, Enzor R, Dey D, He Y, Gehlhausen JR, Lehmann AS, Park SJ, Yang Y, Yang X, et al: The tumor suppressor CDKN3 controls mitosis. J Cell Biol 201: 997-1012, 2013.

12. Song H, Hanlon N, Brown NR, Noble ME, Johnson LN and Barford D: Phosphoprotein-protein interactions revealed by the crystal structure of kinase-associated phosphatase in complex with phosphoCDK2. Mol Cell 7: 615-626, 2001.

13. Yeh CT, Lu SC, Chao CH and Chao ML: Abolishment of the interaction between cyclin-dependent kinase 2 and Cdk-associated protein phosphatase by a truncated KAP mutant. Biochem Biophys Res Commun 305: 311-314, 2003.

14. Wang L, Sun L, Huang J and Jiang M: Cyclin-dependent kinase inhibitor 3 (CDKN3) novel cell cycle computational network between human non-malignancy associated hepatitis/cirrhosis and hepatocellular carcinoma (HCC) transformation. Cell Prolif 44: 291-299, 2011.

15. Barrón EV, Roman-Bassaure E, Sánchez-Sandoval AL, Espinosa AM, Guardado-Estrada M, Medina I, Juárez E, Alfaro A, Bermúdez M, Zamora R, et al: CDKN3 mRNA as a biomarker for survival and therapeutic target in cervical cancer. PLoS One 10: e0137397, 2015.

16. Zang X, Chen M,Zhou Y, Xiao G, Xie Y and Wang X: Identifying CDKN3 gene expression as a prognostic biomarker in lung adenocarcinoma via meta-analysis. Cancer Inform 14 (Suppl 2): S183-S191, 2015.

17. Wang H, Chen H, Zhou H, Yu W and Lu Z: Cyclin-dependent kinase inhibitor 3 promotes cancer cell proliferation and tumorigenesis in nasopharyngeal carcinoma by targeting p27. Oncol Res 25: 1431-1440, 2017.

18. Deng M, Wang J, Chen Y, Zhang L, Xie G, Liu Q, Zhang T, Yuan P and Liu D: Silencing cyclin-dependent kinase inhibitor 3 inhibits the migration of breast cancer cell lines. Mol Med Rep 14: 1523-1530, 2016.

19. Xu CQ, Zhu ST, Wang M, Guo SL, Sun XJ, Cheng R, Xing J, Wang WH, Shao LL and Zhang ST: Pathway analysis of differentially expressed genes in human esophageal squamous cell carcinoma. Eur Rev Med Pharmacol Sci 19: 1652-1661, 2015.

20. Livak KJ and Schmittgen TD: Analysis of relative gene expression data using real-time quantitative PCR and the 2(-Delta Delta C(T)) method. Methods 25: 402-408, 2001.

21. Chandrashekar DS, Bashel B, Balasubramanya SAH, Creighton CJ, Ponce-Rodriguez I, Chakravarthi BVSK and Varambally S: UALCAN: A portal for facilitating tumor subgroup gene expression and survival analyses. Neoplasia 19: 649-658, 2017.

22. Hu N, Clifford RJ, Yang HH, Wang C, Goldstein AM, Ding T, Taylor PR and Lee MP: Genome wide analysis of DNA copy number neutral loss of heterozygosity $(\mathrm{CNNLOH})$ and its relation to gene expression in esophageal squamous cell carcinoma. BMC Genomics 11: 576, 2010.

23. Chen YK, Tung CW, Lee JY, Hung YC, Lee CH, Chou SH, Lin HS, Wu MT and Wu IC: Plasma matrix metalloproteinase 1 improves the detection and survival prediction of esophageal squamous cell carcinoma. Sci Rep 6: 30057, 2016.

24. Chen W, Zheng R, Baade PD, Zhang S, Zeng H, Bray F, Jemal A, $\mathrm{Yu}$ XQ and He J: Cancer statistics in China, 2015. CA Cancer J Clin 66: 115-132, 2016.

25. Lai MW, Chen TC, Pang ST and Yeh CT: Overexpression of cyclin-dependent kinase-associated protein phosphatase enhances cell proliferation in renal cancer cells. Urol Oncol 30: 871-878, 2012.

26. Chang SL, Chen TJ, Lee YE, Lee SW, Lin LC and He HL: CDKN3 expression is an independent prognostic factor and associated with advanced tumor stage in nasopharyngeal carcinoma. Int J Med Sci 15: 992-998, 2018.

27. Li Y, Ji S, Fu LY, Jiang T, Wu D and Meng FD: Knockdown of Cyclin-dependent kinase inhibitor 3 inhibits proliferation and invasion in human gastric cancer cells. Oncol Res 25: 721-731, 2017.

28. Dai W, Miao H, Fang S, Fang T, Chen N and Li M: CDKN3 expression is negatively associated with pathological tumor stage and CDKN3 inhibition promotes cell survival in hepatocellular carcinoma. Mol Med Rep 14: 1509-1514, 2016.

29. Wang J, Che W, Wang W, Su G, Zhen T and Jiang Z: CDKN3 promotes tumor progression and confers cisplatin resistance via RAD51 in esophageal cancer. Cancer Manag Res 11: 3253-3264, 2019.

30. Liu J, Min L, Zhu S, Guo Q, Li H, Zhang Z, Zhao Y, Xu C and Zhang S: Cyclin-dependent kinase inhibitor 3 promoted cell proliferation by driving cell cycle from G1 to S phase in esophageal squamous cell carcinoma. J Cancer 10: 1915-1922, 2019. 